\title{
The double engines and single checkpoint theory of endometriosis
}

\author{
Che-Fang Hsu ${ }^{1}$, Aye Aye Khine ${ }^{1}$, Hsuan-Shun Huang ${ }^{1}$, and Tang-Yuan Chu ${ }^{1}$ \\ ${ }^{1}$ Hualien Tzu Chi Hospital Buddhist Tzu Chi Medical Foundation
}

February 10, 2022

\begin{abstract}
Endometriosis is a common chronic disease of women characterized by ectopic localization of the endometrial tissue in the peritoneal cavity. The pathogenesis of endometriosis closely resembles that of gynecological cancers in ways of menstruationand ovulation-driven, wide intraperitoneal seeding, somatic hypermutation, and immune escape. While retrograde menstruation is regarded as the primary cause of endometriosis, the role of ovulation in the development of endometriosis has long been overlooked. We present the double engines, one checkpoint theory of the evolution of endometriosis. This new theory may lead to new concepts and strategies for preventing and treating this disease.
\end{abstract}

\section{The double engines and single checkpoint theory of endometriosis}

Che-Fang $\mathrm{Hsu}^{1}{ }^{1}$, Khine Aye Aye ${ }^{1,}$, Hsuan-Shun Huang ${ }^{1}$, Tang-Yuan $\mathrm{Chu}^{1,2,3,4^{*}}$

${ }^{1}$ Center for Prevention and Therapy of Gynecological Cancers, Department of Medical Research, Hualien Tzu Chi Hospital, Buddhist Tzu Chi Medical Foundation, Hualien 970, Taiwan, ROC.

${ }^{2}$ Department of Obstetrics \& Gynecology, Hualien Tzu Chi Hospital, Buddhist Tzu Chi Medical Foundation, Hualien 970, Taiwan, ROC.

Running title: A new theory of genesis of endometriosis.

The authors consider that the first two authors should be regarded as joint First Authors equal contribution.

*Author to whom correspondence should be addressed.

Correspondence:

Postal address: Buddhist Tzu Chi General Hospital, 707, Section 3, Chung-Yang Road, Hualien 970, Taiwan.

E-mail address:hidrchu@gmail.com

Tel.: +886-3-8561825

All authors have nothing to disclose.

The research was supported by grants of the Ministry of Science and Technology, Taiwan (MOST 107-2314-B303-013-MY3, MOST 110-2314-B-303-008), and Buddhist Tzu Chi General Hospital, Taiwan (TCMMP10801-01).

\section{ABSTRACT}

Endometriosis is a common chronic disease of women characterized by ectopic localization of the endometrial tissue in the peritoneal cavity. The pathogenesis of endometriosis closely resembles that of gynecological cancers in ways of menstruation- and ovulation-driven, wide intraperitoneal seeding, somatic hypermutation, and immune escape. While retrograde menstruation is regarded as the primary cause of endometriosis, the 
role of ovulation in the development of endometriosis has long been overlooked. We present the double engines, one checkpoint theory of the evolution of endometriosis. This new theory may lead to new concepts and strategies for preventing and treating this disease.

KEYWORDS : endometriosis, ovulation, retrograde menstruation, hypermutation, immune checkpoint

\section{Endometriosis behaves like a cancer of no-kill}

Endometriosis is a disease wherein an endometrium-like tissue grows ectopically outside the uterus. It affects about $10 \%$ of reproductively-active females and causes debilitating dysmenorrhea, pelvic pain, and infertility and also leads to substantial social and healthcare costs (1). The pathobiology underlying this disease is complicated and as yet undeciphered, as it is associated with heterogeneous symptoms and affects multiple physiological systems $(2,3)$. While imaging studies, such as ultrasonography and MRI, can only suggest a possible diagnosis (4) (5) surgical visualization and histological verification can establish a reliable diagnosis. Nevertheless, the pathophysiological mechanisms that lead to the development and maintenance of endometriosis remain unclear. Currently available medical therapies focus on treating the symptoms rather than the causes; thus, efficacy is limited because of severe side effects that prohibit long-term treatment (6). Moreover, endometriosis often recurs after discontinuing treatment. Notably, as endometriosis has cancerlike characteristics, such as hypermutation, intraperitoneal spreading and invasion, and the propensity of its recurrence post-treatment (7), it can be regarded as cancer of no-kill.

\section{Enigmas and missing links in endometriosis}

Although there is no clear evidence on the etiology of endometriosis, there are multiple hypotheses regarding the origin of endometriotic lesions. The most acknowledged hypothesis is that of retrograde menstruation wherein there is a backflow of menstrual blood into the peritoneal cavity through the fallopian tubes (8). Tissue fragments in this retrograde blood flow seed the ectopic sites on the peritoneal and ovarian surfaces. However, retrograde menstruation is almost universal among menstruating women (9), whereas endometriosis is uncommon. Thus, factors such as female sex hormones and inflammatory and immunological milieus may determine the viability and persistence of the endometrial tissue in the ectopic location (6, 9-11). Moreover, recent studies have implied missing links between the development and persistence of endometriosis. In the following sections, we will present three missing links in the pathogenesis of endometriosis and discuss the underlying mechanisms and possible clinical applications in the prevention of and targeted therapy for endometriosis.

\section{Missing link 1: Ovulation is an overlooked risk factor in the development of endometriosis}

\section{1-1. Epidemiological evidence}

Epidemiological studies have revealed multiple risk factors for endometriosis (10) (3) that can be categorized into two categories based on ovulation.

(1) The first category includes risk factors related to incessant ovulation, such as short length of menstrual cycles, nulliparity, short duration of lactation, and no consumption of oral contraceptives (11-14). These risk factors are associated with increased frequency of exposure to the menstrual/ovulation cycle. Moreover, multiple lines of evidence indicate that frequent exposure to both menstrual and ovulation cycles are responsible for the development of endometriosis (detailed in the following section).

(2) The second category includes risk factors associated with exposure to insulin-like growth factor (IGF) axis proteins that are the main effectors of growth hormones (GHs) in pubertal body-height spurt (15). Low body-mass index and waist-to-hip ratio are two GH/IGF-related risk factors for endometriosis (16-18) and are also the two major phenotypes of the GH/IGF axis. The risk of developing endometriosis is reported to be positively associated with a woman's height and negatively associated with a woman's weight (19). Additionally, large-scale genome-wide association studies have reported the positive association of IGF axis genes, including insulin-like growth factor (IGFBP)2, IGFBP3, the IGFBP-cleavage enzyme PAPP-A, and IGF-1R, with adult body height (20). The IGF axis signal is highly abundant and active in the ovulatory 
follicular fluid (FF), which is physiologically important for the regeneration of the damaged tissue post ovulation (21). Reportedly, this ovulatory growth signal also plays an essential role in the genesis of ovarian high-grade serous carcinoma (HGSC), conferring for the stemness activation, clonal expansion and anchorage independent growth phenotypes (detailed in the later section)(21).

\section{1-2. Clinical evidence}

Ovulation as a risk factor for the development of endometriosis can be explained by either repeated exposure to retrograde menstruation, incessant ovulation, or both. While ovulation and menstruation have always been linked together, they can be individually inhibited. In the following paragraphs, we present five lines of clinical evidence indicating the crucial role of ovulation in the development of endometriosis.

(1) Use of combined oral contraceptives to inhibit ovulation while maintaining menstruation reduces the risk of new diagnosis (22) and recurrence of endometriosis (23). Clinically, ovulation is absolutely inhibited, whereas retrograde menstruation, although reduced, persists. Thus, inhibiting ovulation, but not menstruation, seems to affect the development of endometriosis.

(2) A stronger evidence that ovulation plays an essential role in the development of endometriosis is based on another scenario contrary to [1], where only menstruation is inhibited by the levonorgestrel-releasing intrauterine system (Mirena). Release of levonorgestrel at $14 \mu \mathrm{g} / 24 \mathrm{hrs}$ dosage is sufficient to induce endometrial atrophy and amenorrhea, but the systemic concentration of levonorgestrel at $218 \mathrm{ng} / \mathrm{L}$ is insufficient to inhibit ovulation (24). In a randomized trial where Mirena and placebo were tested for long-term maintenance therapy to prevent recurrence of endometriosis, Mirena was found to be ineffective in preventing recurrence of endometriosis (25). Thus, mitigating menstruation when ovulation persists is ineffective in preventing the genesis of endometriosis, leading to recurrence.

(3) Upon removal of the uterus (the source of retrograde menses), it is the presence or absence of the ovary that significantly affects the risk of recurrence of endometriosis post-surgical treatment (26). A meta-analysis comparing hysterectomy-based treatment of endometriosis/adenomyosis to oophorectomy-based treatment, preserving the ovary, reported an eight-fold increase in the risk of reoperation and a six-fold increase in the risk of recurrent pain (27). Thus, loss of both ovulation and sex hormones (estrogen) may be responsible for the prevention of recurrence of endometriosis; however, the successive clinical scenario indicates that estrogen plays a less significant role in the recurrence of endometriosis.

(4) Although gonadotropin-releasing hormone agonist (GnRHa) suppresses all the supposed elements of endometriosis, i.e., ovulation, menstruation, and hormone production, it is only as effective as oral contraceptives are in treating endometriosis (28). Moreover, due to the severe side effects of hormone deprivation, addition of estrogen to GnRHa therapy is commonly practiced. If estrogen is essential for the development of endometriosis, this practice would compromise the effect of GnRHa; however, studies have reported that this addition does not compromise the efficacy of disease control (29-31). Thus, estrogen alone would not support the growth of endometriosis, ovulation and menstruation are also necessary.

(5) Lastly, the exceptionally high efficacy of progesterone-only pills and dienogest in suppressing both ovulation and menstruation further enforces the theory that retrograde menses and ovulation are the major factors that govern development of endometriosis.

\section{1-3. Growth factors released during ovulation may promote the development of endometrio- sis}

Previously, in vitro, in vivo, and clinical research on the etiology of HGSC (high-grade serous carcinoma) has implicated that incessant ovulation (32) (https; (https; (https is responsible for the development of HGSC of the fallopian tube fimbrial epithelium (FTE) (33). Similar to endometriotic lesions, the fimbriae, which pick up oocytes, are exposed to the ovulatory follicular fluid (FF) during and after ovulation. The FF carries growth factors, such as IGF2 and hepatocyte growth factor (HGF), that ensures the regeneration of damaged fimbrial and ovarian epithelium after ovulation and also malignantly transform the fimbrial epithelial cells $(34,35)$. Ovulation thus sources these growth factors into the peritoneal fluid and nourishes 
the endometriosis lesions which express the receptors. In addition to growth factors, a hormonal active ovary also sources the key mitogenic hormone estrogen and differentiation hormone progesterone into the peritoneal fluid (36). Thus, peritoneal fluid is regarded as an ovarian exudate constituted by the secretion products of ovulation $(37,38)$. In another word, ovulation can be an engine that powers the growth of endometriosis in the peritoneal cavity.

\section{1-4. Mechanism of ovulation-induced clonal expansion and cell proliferation}

The mechanism by which the $\mathrm{FF}$ promotes tissue regeneration and malignant transformation has been elucidated recently $(21,39)$. As summarized in Figure 1, two growth-related signaling pathways are involved. IGF signaling pathway is responsible for stemness activation and clonal expansion, whereas HGF signaling pathway is responsible for cell proliferation, migration, and invasion. In brief, the FF harbors abundant IGF2 molecules that are trapped after binding to IGFBP2 and IGFBP6. Upon ovulation, PAPP-A is activated after engaging with glycosaminoglycans on the FTE and cleaves IGF2, which acts on its membrane receptor IGF-1R. Subsequently, FF-IGF activates stemness and clonal expansion as well as malignant transformation through the AKT/NANOG pathway and the AKT/mTOR pathway (21). Since the cyclic ovarian activity is the main source of peritoneal fluid, which bath the endometriosis lesions.

\section{Activation of the HGF in the FF sustains cell transformation-based activities after ovulation}

The HGF is the second main growth-related component in the FF. Upon binding to the c-MET receptor on the FTE, the HGF promotes activities related to malignant transformation, including promotion of cell proliferation, migration, invasion, and anchorage-independent growth. Unlike the IGF axis, which functions transiently after ovulation, activity of the HGF in ovulatory FF is sustained long after the release of an ovum into the peritoneal fluid and throughout the menstrual cycle as well. Mechanistically, ovulatory injury activates the extrinsic coagulation cascade, in which activated thrombin cleaves the pro-HGFA to activatedHGFA that subsequently cleaves the pro-HGF to form HGF. The FF has a high reserve of these coagulation cascade proteins and HGFA/HGF. The cascade is activated upon injuries to the ovaries and has a continuous activity in the peritoneal fluid. Thus, c-MET-expressing tissues, including the FTE and the endometriosis lesions in pelvic cavity, are activated and exhibit growth and invasion phenotypes (40). Reportedly, c-MET, like IGF-1R, is highly expressed in the eutopic and the ectopic endometrial tissues $(41,42)$. Their signals promote the proliferation and invasion of the endometrial epithelial cells and stromal cells $(41,43,44)$ playing important roles in the development of endometriosis and associated pelvic pain $(44,45)$ (Figure 2).

\section{High concentrations of soluble extracellular matrix (ECM) proteins in the FF may also con- tribute to the development of endometriosis}

In addition to the IGF-axis proteins and the HGF, extremely high concentrations of the ECM proteins are present in ovulatory FF (46). This leads to a steep concentration gradient between the FF, peritoneal fluid $(\mathrm{PF})$, and serum (47). The follicle-blood barrier (FBB) between the granulosa cells lining the ovarian follicle and the surrounding theca cells traps molecules larger than $300 \mathrm{kDa}$ in the ovarian follicle $(48,49)$. As shown in Table 1, the FF contains extraordinarily high concentrations of macromolecule-bound growth factors, sex hormones, and soluble ECM proteins (46). Estrogen is the most concentrated molecule in the FF (990-fold and 2900-fold higher in the FF than in the PF and serum) and is essential for the initiation and development of endometriosis (50). In addition, concentrations of PAPP-A and HGF are 36-fold and 80-fold higher in the FF than in the PF, respectively; PAPP-A has a 500-fold higher concentration in the FF than in serum (Table 1 ). We found that PAPP-A, which cleaves IGFBP $2 / 6$, can release IGF2 to bind with its receptor IGF-1R on FTEs and is positively associated with the cell transformation activities of the FF (21). Soluble fibronectin and laminin are the 4th and 69th most abundant proteins in the FF (46). They promote cell adhesion, seeding, migration, and invasion in different cancers, including ovarian cancer, through the integrins $\alpha v \beta 1$ and $\alpha \mathrm{v} \beta 3(51,52)$. Their levels in the FF are approximately 100 -fold higher than that in serum. Other soluble ECM proteins, such as vitronectin (the 11th) (53), serpin family D member 1 (SERPIND1) (the 38th) (54), and periostin (the 112th) (55), present in the FF also exhibit different oncogenic activities in ovarian cancer. The SERPIND1 protein belongs to a family of serine protease inhibitors, and its high expression in ovarian 
cancer tissues confers poor patient prognoses (54). It promotes ovarian cancer cell proliferation, epithelialto-mesenchymal transition, migration, and invasion and inhibits apoptosis via the PI3K/AKT pathway (54). Moreover, vitronectin promotes adhesion and chemoattraction in ovarian cancer cells via the $\alpha 5 \beta 1$ and $\alpha \mathrm{v} \beta 3$ integrins and urokinase plasminogen activator surface receptor (53). Whereas, periostin, overexpressed in advanced and recurrent ovarian cancer, promotes adhesion, migration, and invasion in ovarian cancer and human umbilical vein endothelial cells. It also promotes angiogenesis and xenograft metastasis when overexpressed in HGSC cells (56). However, the levels of vitronectin and periostin in tissue fluids and their role in early tubal carcinogenesis and development of endometriosis are unknown.

\section{Missing link 2: Retrograde menstruation is the source of the tissue-of-origin in endometriosis and also augments the disease}

The human menstrual cycle is an orchestrated series of physiological, cellular, and biochemical changes that are complexly regulated by hormones and involves the routine thickening of the endometrium in preparation for embryo implantation. Menstruation is the cyclic breakdown of the endometrium in the absence of an embryo implantation, and it is one of the three (other than ovulation and parturition) physiological tissue injuries caused by acute inflammation (57). Unexpectedly, $90 \%$ of all women who underwent laparoscopy during menstruation had a bloody peritoneal fluid, suggesting that retrograde menstruation is a common phenomenon in women (9). This provides the basis of Sampson's theory of the genesis of endometriosis which states that the eutopic endometrium is seeded into the peritoneal cavity via retrograde menstruation (8).

Since vigorous regeneration of the endometrial tissue is required at each menstruation, the shed endometrial tissue in the menstrual flow are very likely to harbor stem/progenitor cells. It is up to these stem cells to establish the ectopic growth and development of the endometriosis. Meanwhile, the regenerative capacity of the endometrium is attributed to stem or progenitor cells residing in the basalis layer which is not shed by the menstruation, and providing a source to regenerate the functionalis layer each month (58). In the eutopic endometrium of endometriosis women, there is a propensity for localization of stem cell markerpositive basalis-like cells in the functional is layer (59). Also, the menstrual blood of endometriosis women also tends to harbor fragments of shed basalis tissue.

Mechanisms involving the survival and growth of endometrial stem cells in the peritoneal environment are crucial for the development of endometriosis. These mechanisms involve (1) overcoming the apoptosis due to loss of attachment (or anoikis resistance) after intraperitoneal shedding, (2) attachment and proliferation on the peritoneum, (3) angiogenesis, and (4) stromal invasion. This stepwise seeding and metastasis follows a mechanism identical to intraperitoneal metastasis observed in ovarian HGSC. Moreover, patients with endometriosis had a significantly increased risk of e upper genital tract and peritoneal infections (60). The microbiome and oxidative stress introduced by the retrograde menstruation may cause microtrauma to the mesothelium lining, exposing the intercellular matrix and inducing acute inflammation(61). These changes may contribute to the successful implantation of the ectopic endometrial tissues.

\section{Extrauterine menstruation in endometriosis triggers inflammatory response}

In addition to being the source of ectopic endometrial tissue, menstrual debris in the peritoneal cavity readily release damage-associated molecular patterns (DAMPs) that trigger innate immunity in women with or without endometriosis (62). The DAMPs released by activated mast cells and macrophages, lead to the secretion of pro-inflammatory and pro-nociceptive mediators, exacerbating inflammation and generating pain signals in endometriosis; thus, the symptoms of peritoneal inflammation persist and strengthen (63).

In retrograde menstruation, erythrocytes and their debris are engulfed by macrophages, inducing oxidative stress (64). Activated macrophages play an essential role in the degradation of erythrocytes and release of pro-oxidative and pro-inflammatory factors, such as heme and iron (65). Indeed, higher levels of iron, ferritin, and hemoglobin have been found in the peritoneal fluid of women with endometriosis than in those without (65). Release of iron and reactive oxygen species (ROS) has been implicated in the formation of deleterious free radicles where ferrous ion catalyzes the Fenton reaction, causing oxidative injury to cells 
(65). Thus, ROS may also help the destruction of the peritoneal mesothelium, allowing the attachment and growth of ectopic endometrial cells in the peritoneal cavity (66).

Similar to the mechanism of regeneration after ovulation, HGF is strongly expressed in the human endometrial epithelium during menstruation, stimulating proliferation, migration, and lumen formation in human endometrial epithelial cells $(67,68)$. Studies have reported that HGF levels in the PF are elevated in women with endometriosis (69). In addition, the IGF axis also plays an essential role in endometrial biology, with IGFs being secreted by the stroma and their receptors being expressed by the endometrial epithelium (70). These components of the regenerating machinery may be released into the peritoneal cavity from retrograde menses and help in the initiation and progression of endometriosis.

\section{Missing link 3: Hypermutation and immune escape in endometriosis}

\section{Hypermutation phenotype of endometriosis}

After menstrual shedding, the body vigorously regenerates the endometrium by activating and recruiting local (endometrial) (71) and bone marrow-derived (72) stem cells to the uterine lining, followed by asymmetric cell division and clonal expansion. However, DNA replication during cell division is not without error. Each round of replication creates thousands of DNA mismatches in the genome. These mismatches are repaired by the DNA mismatch repair pathway with $99.9 \%$ efficacy, leaving a tolerable error rate of fewer than ten mutations per replication per genome (73). However, incessant regeneration of the endometrial tissue causes replication errors to accumulate in stem cells. In the asymmetric division of stem cells, one daughter cell is designated to differentiate and eventually shed with menstruation, whereas the other daughter cell retains stemness and passes the mutation indefinitely. With each subsequent menstruation, additional mutations accumulate in the stem cells and their progenies in the endometrium and the endometriosis. Recently, wholegenome sequencing of 292 endometrial glands microdissected from 28 women detected a high mutation load of 1521 base substitutions (ranging from 209-2833) per person (74). This number was associated with the age of a woman, with a linear accumulation of 29 bp substitutions per gland per year. Nevertheless, a much higher mutation load was observed in both eutopic and ectopic endometrial cells obtained from women with endometriosis, $\sim 25$ mutations per megabase (75), than that in women without endometriosis. This high mutation load surpassed that of common cancers $(76,77)$ and was similar to that of the ultra-mutated group of endometrial cancer patients (78). Interestingly, although these patients had a similar high somatic mutation load in eutopic and ectopic endometrial cells, the latter carried a higher mutated allele frequency in cancer-driving genes $(79,80)$, indicating a cancer-like evolution with clonal selection.

\section{Immune escape in endometriosis and the possibility of immune checkpoint blockade therapy}

Expression of neoantigens due to hypermutation renders the mutant cells susceptible to the cytotoxic effects of immune surveillance. One crucial mechanism of this surveillance is to identify normal, orthotopic, and hardly mutated "self" cells and to eradicate hypermutated and ectopically-localizing "non-self" cells by activation of cytotoxic T cells. This identification occurs through the T cell receptor (TCR)/antigen/MHC self-recognition complex and the PD-1/PD-L1 immune checkpoint, a barrier to check the immune surveillance. Once a cell is recognized as "self" by the TCR/MHC complex, the PD-L1 ligand of the "self" cell binds to the PD1 receptor on the $\mathrm{T}$ cell, suppressing $\mathrm{T}$ cell-mediated cytotoxicity and leading to immune tolerance. In contrast, neoantigen-carrying cancer cells not recognized as "self" undergo T cell-mediated cytotoxicity and bypass the immune checkpoint (81). Given the same hyper- and ultra-mutation, we predict that the PD$1 /$ PDL1 immune checkpoint is also turned on during the development of endometriosis, thus conferring immune tolerance. Figure 3 demonstrated an example of overexpression of PD1 and PD-L1 in both eutopic endometrium and ectopic endometrial lesions in an endometriosis patient (Figure 3), concurring with a previous report (82). Thus, this can also predict the efficacy of immune checkpoint blockade in treating endometriosis. Moreover, testing the DNA mutation load in the eutopic endometrium can be a potential biomarker to predict the therapeutic response of a patient with endometriosis, similar to the documented practices in treating patients with hyper- or ultra-mutated endometrial cancers (83).

In addition to the immune checkpoint, loss of progesterone activity or progesterone resistance is also involved 
in the impairement of immune surveillance. Women with endometriosis exhibit reduced endometrial response to progesterone during the secretory phase of the menstrual cycle (34). Since the anti-inflammatory action of progesterone is required for normal immune cell migration and function across the menstrual cycle, loss of normal progesterone activity may contribute to changes in the immune physiology of both the uterus and the peritoneal cavity, as noted in patients with endometriosis (16).

\section{Implications in pathogenesis and clinical management}

One curiosity would be whether the ovulation engine drives the initial implant or the progression of endometriosis. Currently, there is no direct evidence to answer it. However, given the stemness activation and clonal expansion activity of IGF2 and the clonogenicity activity of HGF in FF, we predict ovulation would facilitate both the initiation and progression of endometriosis and are currently conduction animal studies to answer it.

The double engines and single checkpoint theory also provides guidance on how to manage and how to prevent the recurrence of endometriosis. All the three pillars, i.e., ovulation, menstruation and progesterone insensitivity are overcome during pregnancy. Thus, pregnancy is the best drug for endometriosis. Furthermore, the theory also firmly supports conventional medications for treating endometriosis by inhibiting ovulation and reducing menstruation. The efficacy of the inexpensive and well-tolerable oral contraceptives and that of the expensive GnRH agonist and antagonists (Elagolix) can all be based on the inhibition of ovulation and menstruation (84). Progestin preparations that target both ovulation and menstruation, such as dienogest (Vissane) and desogestrel (Marvelon) that are licensed for treating endometriosis and for contraception, respectively, are currently the most effective non-invasive therapeutics for controlling and preventing the recurrence of endometriosis $(85,86)$. Moreover, the inherent anti-inflammatory and anti-estrogenic activities of progesterone enforce its effectiveness (87). Meanwhile, the well-established local progestin preparation, Mirena, works efficiently in inhibiting menstruation but does not affect pituitary function and ovulation due to its low systemic distribution (88). Although Mirena shows equal efficacy as a GnRHa in relieving endometriosis-associated pain (89), it is not effective in preventing the recurrence of ovarian endometrioma (25).

The "checkpoint" in the proposed theory, i.e., the establishment of immune tolerance via the immune checkpoint, also provides PD1 and PDL1 as a target for immune checkpoint blockade for treatment of endometriosis. While immune checkpoint inhibitors have been licensed for the treatment of a wide range of cancers that are characterized by genomic hypermutation and high replication error rate (87), endometriosis with identical phenotypes may very likely respond similarly to the same treatment. Identical tissue biomarkers, PD-L1 expression in tissue sections and mismatch repair deficiency (identified by microsatellite instability) and mutation load (identified by next-generation sequencing) in tissue DNA, can be used to predict the response and selection of eligible cases (90). A long-lasting response can be expected because of the presence of active immunological memory cells (91).

\section{Conclusion:}

The enigma of the pathogenesis of endometriosis can largely be explained by the proposed double engines and single checkpoint theory that consists of three pillars: (1) Ovulatory FF contains growth and remodeling factors that promote the genesis and development of endometriosis. (2) Retrograde menstrual flow seeds ectopic endometrial tissue to the peritoneal cavity and triggers inflammation that promotes the severity of disease. (3) Turning on of the immune checkpoint and lowing progesterone activity allows evasion of immune surveillance. These pillars, as summarized in Figure 4, explain the pathogenesis of endometriosis. The new theory may open new opportunities and strategies for the prevention and management of the disease.

\section{Author Contributions}

Conceptualization: T.-Y. Chu and C.-F. Hsu; data curation: T.-Y. Chu and C.-F. Hsu; drafting and revising the manuscript: A.A. Khine and T.-Y. Chu; supervision: T.-Y. Chu.; C.-F. Hsu and H.-S. Huang completed relevant preliminary studies. All authors have read and agreed to the published version of the manuscript. 


\section{Conflicts of Interest}

The authors declare no conflict of interest.

\section{Reference}

1. Zondervan KT, Becker CM, Missmer SA. Endometriosis. N Engl J Med. 2020;382(13):1244-56.

2. Koninckx PR, Ussia A, Adamyan L, Wattiez A, Gomel V, Martin DC. Heterogeneity of endometriosis lesions requires individualisation of diagnosis and treatment and a different approach to research and evidence based medicine. Facts Views Vis Obgyn. 2019;11(1):57-61.

3. Smolarz B, Szyllo K, Romanowicz H. Endometriosis: Epidemiology, Classification, Pathogenesis, Treatment and Genetics (Review of Literature). Int J Mol Sci. 2021;22(19).

4. Agarwal SK, Chapron C, Giudice LC, Laufer MR, Leyland N, Missmer SA, et al. Clinical diagnosis of endometriosis: a call to action. Am J Obstet Gynecol. 2019;220(4):354 e1- e12.

5. Koninckx PR, Deslandes A, Ussia A, Di Giovanni A, Hanan G, Tahlak M, et al. Preoperative imaging of deep endometriosis: pitfalls of a diagnostic test before surgery. Facts Views Vis Obgyn. 2021;12(4):265-71.

6. Zondervan KT, Becker CM, Koga K, Missmer SA, Taylor RN, Vigano P. Endometriosis. Nat Rev Dis Primers. 2018;4(1):9.

7. Guo SW. Cancer-associated mutations in endometriosis: shedding light on the pathogenesis and pathophysiology. Hum Reprod Update. 2020;26(3):423-49.

8. Sampson JA. Metastatic or Embolic Endometriosis, due to the Menstrual Dissemination of Endometrial Tissue into the Venous Circulation. Am J Pathol. 1927;3(2):93-110 43.

9. Halme J, Hammond MG, Hulka JF, Raj SG, Talbert LM. Retrograde menstruation in healthy women and in patients with endometriosis. Obstet Gynecol. 1984;64(2):151-4.

10. Shafrir AL, Farland LV, Shah DK, Harris HR, Kvaskoff M, Zondervan K, et al. Risk for and consequences of endometriosis: A critical epidemiologic review. Best Pract Res Clin Obstet Gynaecol. 2018;51:1-15.

11. Missmer SA, Hankinson SE, Spiegelman D, Barbieri RL, Malspeis S, Willett WC, et al. Reproductive history and endometriosis among premenopausal women. Obstet Gynecol. 2004;104(5 Pt 1):965-74.

12. Sangi-Haghpeykar H, Poindexter AN, 3rd. Epidemiology of endometriosis among parous women. Obstet Gynecol. 1995;85(6):983-92.

13. Berube S, Marcoux S, Maheux R. Characteristics related to the prevalence of minimal or mild endometriosis in infertile women. Canadian Collaborative Group on Endometriosis. Epidemiology. 1998;9(5):504-10.

14. Kavoussi SK, Odenwald KC, As-Sanie S, Lebovic DI. Incidence of ovarian endometrioma among women with peritoneal endometriosis with and without a history of hormonal contraceptive use. Eur J Obstet Gynecol Reprod Biol. 2017;215:220-3.

15. Dixit M, Poudel SB, Yakar S. Effects of GH/IGF axis on bone and cartilage. Mol Cell Endocrinol. 2021;519:111052.

16. Schmitz KH, Xie D, Teal V, Ballard-Barbash R, Berrigan D. Association of IGF axis hormones with waist-to-hip ratio varies by physical activity. In Vivo. 2011;25(2):245-50.

17. Gram IT, Norat T, Rinaldi S, Dossus L, Lukanova A, Tehard B, et al. Body mass index, waist circumference and waist-hip ratio and serum levels of IGF-I and IGFBP-3 in European women. Int J Obes (Lond). 2006;30(11):1623-31. 
18. Lafay Pillet MC, Schneider A, Borghese B, Santulli P, Souza C, Streuli I, et al. Deep infiltrating endometriosis is associated with markedly lower body mass index: a 476 case-control study. Hum Reprod. $2012 ; 27(1): 265-72$.

19. Signorello LB, Harlow BL, Cramer DW, Spiegelman D, Hill JA. Epidemiologic determinants of endometriosis: a hospital-based case-control study. Ann Epidemiol. 1997;7(4):267-741.

20. Lango Allen H, Estrada K, Lettre G, Berndt SI, Weedon MN, Rivadeneira F, et al. Hundreds of variants clustered in genomic loci and biological pathways affect human height. Nature. 2010;467(7317):832-8.

21. Hsu CF, Huang HS, Chen PC, Ding DC, Chu TY. IGF-axis confers transformation and regeneration of fallopian tube fimbria epithelium upon ovulation. EBioMedicine. 2019;41:597-609.

22. Vercellini P, Eskenazi B, Consonni D, Somigliana E, Parazzini F, Abbiati A, et al. Oral contraceptives and risk of endometriosis: a systematic review and meta-analysis. Hum Reprod Update. 2011;17(2):159-70.

23. Muzii L, Di Tucci C, Achilli C, Di Donato V, Musella A, Palaia I, et al. Continuous versus cyclic oral contraceptives after laparoscopic excision of ovarian endometriomas: a systematic review and metaanalysis. Am J Obstet Gynecol. 2016;214(2):203-11.

24. Apter D, Gemzell-Danielsson K, Hauck B, Rosen K, Zurth C. Pharmacokinetics of two low-dose levonorgestrel-releasing intrauterine systems and effects on ovulation rate and cervical function: pooled analyses of phase II and III studies. Fertil Steril. 2014;101(6):1656-62 e1-4.

25. Chen YJ, Hsu TF, Huang BS, Tsai HW, Chang YH, Wang PH. Postoperative maintenance levonorgestrelreleasing intrauterine system and endometrioma recurrence: a randomized controlled study. Am J Obstet Gynecol. 2017;216(6):582 e1- e9.

26. Namnoum AB, Hickman TN, Goodman SB, Gehlbach DL, Rock JA. Incidence of symptom recurrence after hysterectomy for endometriosis. Fertil Steril. 1995;64(5):898-902.

27. Rizk B, Fischer AS, Lotfy HA, Turki R, Zahed HA, Malik R, et al. Recurrence of endometriosis after hysterectomy. Facts Views Vis Obgyn. 2014;6(4):219-27.

28. Vercellini P, Trespidi L, Colombo A, Vendola N, Marchini M, Crosignani PG. A gonadotropin-releasing hormone agonist versus a low-dose oral contraceptive for pelvic pain associated with endometriosis. Fertil Steril. 1993;60(1):75-9.

29. Hornstein MD, Surrey ES, Weisberg GW, Casino LA. Leuprolide acetate depot and hormonal add-back in endometriosis: a 12-month study. Lupron Add-Back Study Group. Obstet Gynecol. 1998;91(1):16-24.

30. Surrey ES. Gonadotropin-releasing hormone agonist and add-back therapy: what do the data show? Curr Opin Obstet Gynecol. 2010;22(4):283-8.

31. Surrey ES. Add-back therapy and gonadotropin-releasing hormone agonists in the treatment of patients with endometriosis: can a consensus be reached? Add-Back Consensus Working Group. Fertil Steril. 1999;71(3):420-4.

32. Fathalla MF. Incessant ovulation and ovarian cancer - a hypothesis re-visited. Facts Views Vis Obgyn. 2013;5(4):292-7.

33. Wu NY, Fang C, Huang HS, Wang J, Chu TY. Natural history of ovarian high-grade serous carcinoma from time effects of ovulation inhibition and progesterone clearance of p53-defective lesions. Mod Pathol. 2020;33(1):29-37.

34. Huang HS, Chu SC, Hsu CF, Chen PC, Ding DC, Chang MY, et al. Mutagenic, surviving and tumorigenic effects of follicular fluid in the context of p53 loss: initiation of fimbria carcinogenesis. Carcinogenesis. 2015;36(11):1419-28. 
35. Huang HS, Hsu CF, Chu SC, Chen PC, Ding DC, Chang MY, et al. Haemoglobin in pelvic fluid rescues Fallopian tube epithelial cells from reactive oxygen species stress and apoptosis. J Pathol. 2016;240(4):48494.

36. Koninckx PR, Ussia A, Adamyan L, Gomel V, Martin DC. Peritoneal fluid progesterone and progesterone resistance in superficial endometriosis lesions. Hum Reprod. 2021.

37. Koninckx PR, Renaer M, Brosens IA. Origin of peritoneal fluid in women: an ovarian exudation product. Br J Obstet Gynaecol. 1980;87(3):177-83.

38. Koninckx PR, Heyns W, Verhoeven G, Van Baelen H, Lissens WD, De Moor P, et al. Biochemical characterization of peritoneal fluid in women during the menstrual cycle. J Clin Endocrinol Metab. 1980;51(6):1239-44.

39. Hsu CF, Chen PC, Seenan V, Ding DC, Chu TY. Ovulatory Follicular Fluid Facilitates the Full Transformation Process for the Development of High-Grade Serous Carcinoma. Cancers (Basel). 2021;13(3).

40. Zhang Y, Xia M, Jin K, Wang S, Wei H, Fan C, et al. Function of the c-Met receptor tyrosine kinase in carcinogenesis and associated therapeutic opportunities. Mol Cancer. 2018;17(1):45.

41. Zhou Y, Zeng C, Li X, Wu PL, Yin L, Yu XL, et al. IGF-I stimulates ERbeta and aromatase expression via IGF1R/PI3K/AKT-mediated transcriptional activation in endometriosis. J Mol Med (Berl). 2016;94(8):887-97.

42. Khan KN, Masuzaki H, Fujishita A, Kitajima M, Sekine I, Ishimaru T. Immunoexpression of hepatocyte growth factor and c-Met receptor in the eutopic endometrium predicts the activity of ectopic endometrium. Fertil Steril. 2003;79(1):173-81.

43. Li M, Xin X, Wu T, Hua T, Wang H. HGF and c-Met in pathogenesis of endometrial carcinoma. Front Biosci (Landmark Ed). 2015;20:635-43.

44. Yoshida S, Harada T, Mitsunari M, Iwabe T, Sakamoto Y, Tsukihara S, et al. Hepatocyte growth factor/Met system promotes endometrial and endometriotic stromal cell invasion via autocrine and paracrine pathways. J Clin Endocrinol Metab. 2004;89(2):823-32.

45. Forster R, Sarginson A, Velichkova A, Hogg C, Dorning A, Horne AW, et al. Macrophage-derived insulinlike growth factor-1 is a key neurotrophic and nerve-sensitizing factor in pain associated with endometriosis. FASEB J. 2019;33(10):11210-22.

46. Zamah AM, Hassis ME, Albertolle ME, Williams KE. Proteomic analysis of human follicular fluid from fertile women. Clin Proteomics. 2015;12(1):5.

47. Donnez J, Langerock S, Thomas K. Peritoneal fluid volume and 17 beta-estradiol and progesterone concentrations in ovulatory, anovulatory, and postmenopausal women. Obstet Gynecol. 1982;59(6):687-92.

48. Siu MK, Cheng CY. The blood-follicle barrier (BFB) in disease and in ovarian function. Adv Exp Med Biol. 2012;763:186-92.

49. Shalgi R, Kraicer P, Rimon A, Pinto M, Soferman N. Proteins of human follicular fluid: the blood-follicle barrier. Fertil Steril. 1973;24(6):429-34.

50. Plaino L, Stomati M, Casarosa E, Artini PG, Santuz M, D'Ambrogio G, et al. Ovarian follicular fluid contains immunoreactive estriol: lack of correlation with estradiol concentrations. Gynecol Endocrinol. 2000;14(4):231-5.

51. Yousif NG. Fibronectin promotes migration and invasion of ovarian cancer cells through up-regulation of FAK-PI3K/Akt pathway. Cell Biol Int. 2014;38(1):85-91.

52. Sengupta S, Xiao YJ, Xu Y. A novel laminin-induced LPA autocrine loop in the migration of ovarian cancer cells. FASEB J. 2003;17(11):1570-2. 
53. Schneider G, Suszynska M, Kakar S, Ratajczak MZ. Vitronectin in the ascites of human ovarian carcinoma acts as a potent chemoattractant for ovarian carcinoma: Implication for metastasis by cancer stem cells. J Cancer Stem Cell Res. 2016;4.

54. Guo Q, Zhu L, Wang C, Wang S, Nie X, Liu J, et al. SERPIND1 Affects the Malignant Biological Behavior of Epithelial Ovarian Cancer via the PI3K/AKT Pathway: A Mechanistic Study. Front Oncol. 2019;9:954.

55. Gonzalez-Gonzalez L, Alonso J. Periostin: A Matricellular Protein With Multiple Functions in Cancer Development and Progression. Front Oncol. 2018;8:225.

56. Zhu M, Fejzo MS, Anderson L, Dering J, Ginther C, Ramos L, et al. Periostin promotes ovarian cancer angiogenesis and metastasis. Gynecol Oncol. 2010;119(2):337-44.

57. Critchley HOD, Maybin JA, Armstrong GM, Williams ARW. Physiology of the Endometrium and Regulation of Menstruation. Physiol Rev. 2020;100(3):1149-79.

58. Gargett CE, Nguyen HP, Ye L. Endometrial regeneration and endometrial stem/progenitor cells. Rev Endocr Metab Disord. 2012;13(4):235-51.

59. Hapangama DK, Drury J, Da Silva L, Al-Lamee H, Earp A, Valentijn AJ, et al. Abnormally located SSEA1+/SOX9+ endometrial epithelial cells with a basalis-like phenotype in the eutopic functionalis layer may play a role in the pathogenesis of endometriosis. Hum Reprod. 2019;34(1):56-68.

60. Koninckx PR, Ussia A, Tahlak M, Adamyan L, Wattiez A, Martin DC, et al. Infection as a potential cofactor in the genetic-epigenetic pathophysiology of endometriosis: a systematic review. Facts Views Vis Obgyn. 2019;11(3):209-16.

61. Greaves E, Cousins FL, Murray A, Esnal-Zufiaurre A, Fassbender A, Horne AW, et al. A novel mouse model of endometriosis mimics human phenotype and reveals insights into the inflammatory contribution of shed endometrium. Am J Pathol. 2014;184(7):1930-9.

62. Kobayashi H, Higashiura Y, Shigetomi H, Kajihara H. Pathogenesis of endometriosis: the role of initial infection and subsequent sterile inflammation (Review). Mol Med Rep. 2014;9(1):9-15.

63. Laux-Biehlmann A, d'Hooghe T, Zollner TM. Menstruation pulls the trigger for inflammation and pain in endometriosis. Trends Pharmacol Sci. 2015;36(5):270-6.

64. Van Langendonckt A, Casanas-Roux F, Donnez J. Oxidative stress and peritoneal endometriosis. Fertil Steril. 2002;77(5):861-70.

65. Defrere S, Lousse JC, Gonzalez-Ramos R, Colette S, Donnez J, Van Langendonckt A. Potential involvement of iron in the pathogenesis of peritoneal endometriosis. Mol Hum Reprod. 2008;14(7):377-85.

66. Lousse JC, Van Langendonckt A, Defrere S, Ramos RG, Colette S, Donnez J. Peritoneal endometriosis is an inflammatory disease. Front Biosci (Elite Ed). 2012;4:23-40.

67. Tanaka T, Mizuno K, Umesaki N, Ogita S. Suppressed apoptotic susceptibility in human endometrial epithelial cells pretreated with hepatocyte growth factor. Clin Exp Obstet Gynecol. 1998;25(4):125-8.

68. Sugawara J, Fukaya T, Murakami T, Yoshida H, Yajima A. Hepatocyte growth factor stimulated proliferation, migration, and lumen formation of human endometrial epithelial cells in vitro. Biol Reprod. 1997;57(4):936-42.

69. Godbey WT, Wu KK, Mikos AG. Size matters: molecular weight affects the efficiency of poly(ethylenimine) as a gene delivery vehicle. J Biomed Mater Res. 1999;45(3):268-75.

70. Zhou J, Dsupin BA, Giudice LC, Bondy CA. Insulin-like growth factor system gene expression in human endometrium during the menstrual cycle. J Clin Endocrinol Metab. 1994;79(6):1723-34. 
71. Lv H, Hu Y, Cui Z, Jia H. Human menstrual blood: a renewable and sustainable source of stem cells for regenerative medicine. Stem Cell Res Ther. 2018;9(1):325.

72. Lee YJ, Yi KW. Bone marrow-derived stem cells contribute to regeneration of the endometrium. Clin Exp Reprod Med. 2018;45(4):149-53.

73. Pecina-Slaus N, Kafka A, Salamon I, Bukovac A. Mismatch Repair Pathway, Genome Stability and Cancer. Front Mol Biosci. 2020;7:122.

74. Moore L, Leongamornlert D, Coorens THH, Sanders MA, Ellis P, Dentro SC, et al. The mutational landscape of normal human endometrial epithelium. Nature. 2020;580(7805):640-6.

75. Li X, Zhang Y, Zhao L, Wang L, Wu Z, Mei Q, et al. Whole-exome sequencing of endometriosis identifies frequent alterations in genes involved in cell adhesion and chromatin-remodeling complexes. Hum Mol Genet. 2014;23(22):6008-21.

76. Greenman C, Stephens P, Smith R, Dalgliesh GL, Hunter C, Bignell G, et al. Patterns of somatic mutation in human cancer genomes. Nature. 2007;446(7132):153-8.

77. Pleasance ED, Cheetham RK, Stephens PJ, McBride DJ, Humphray SJ, Greenman CD, et al. A comprehensive catalogue of somatic mutations from a human cancer genome. Nature. 2010;463(7278):191-6.

78. Cancer Genome Atlas Research N, Kandoth C, Schultz N, Cherniack AD, Akbani R, Liu Y, et al. Integrated genomic characterization of endometrial carcinoma. Nature. 2013;497(7447):67-73.

79. Anglesio MS, Papadopoulos N, Ayhan A, Nazeran TM, Noe M, Horlings HM, et al. Cancer-Associated Mutations in Endometriosis without Cancer. N Engl J Med. 2017;376(19):1835-48.

80. Noe M, Ayhan A, Wang TL, Shih IM. Independent development of endometrial epithelium and stroma within the same endometriosis. J Pathol. 2018;245(3):265-9.

81. Sunshine J, Taube JM. PD-1/PD-L1 inhibitors. Curr Opin Pharmacol. 2015;23:32-8.

82. Wu L, Lv C, Su Y, Li C, Zhang H, Zhao X, et al. Expression of programmed death-1 (PD-1) and its ligand PD-L1 is upregulated in endometriosis and promoted by 17beta-estradiol. Gynecol Endocrinol. 2019;35(3):251-6.

83. Green AK, Feinberg J, Makker V. A Review of Immune Checkpoint Blockade Therapy in Endometrial Cancer. Am Soc Clin Oncol Educ Book. 2020;40:1-7.

84. Finley CM, Thompson JR, Bradford GE. Age-parity-season adjustment factors for milk and fat yields of dairy goats. J Dairy Sci. 1984;67(8):1868-72.

85. Vercellini P, Buggio L, Frattaruolo MP, Borghi A, Dridi D, Somigliana E. Medical treatment of endometriosis-related pain. Best Pract Res Clin Obstet Gynaecol. 2018;51:68-91.

86. Andres Mde P, Lopes LA, Baracat EC, Podgaec S. Dienogest in the treatment of endometriosis: systematic review. Arch Gynecol Obstet. 2015;292(3):523-9.

87. Nishihira H, Kigasawa H. Growth of human erythroid and erythroid-granulocytic colonies in culture without addition of exogeneous erythropoietin. Br J Haematol. 1981;49(4):563-6.

88. Nilsson CG, Lahteenmaki P, Luukkainen T. Levonorgestrel plasma concentrations and hormone profiles after insertion and after one year of treatment with a levonorgestrel-IUD. Contraception. 1980;21(3):225-33.

89. Petta CA, Ferriani RA, Abrao MS, Hassan D, Rosa ESJC, Podgaec S, et al. Randomized clinical trial of a levonorgestrel-releasing intrauterine system and a depot GnRH analogue for the treatment of chronic pelvic pain in women with endometriosis. Hum Reprod. 2005;20(7):1993-8. 
90. Eiden LE, Rausch DM, da Cunha A, Murray EA, Heyes M, Sharer L, et al. AIDS and the central nervous system. Examining pathobiology and testing therapeutic strategies in the SIV-infected rhesus monkey. Ann N Y Acad Sci. 1993;693:229-44.

91. Principe N, Kidman J, Goh S, Tilsed CM, Fisher SA, Fear VS, et al. Tumor Infiltrating Effector Memory Antigen-Specific CD8(+) T Cells Predict Response to Immune Checkpoint Therapy. Front Immunol. 2020;11:584423.

92. Rezabek K, Moosova M, Pavelkova J, Moos J, Filova V. [Follicular fluid and serum concentrations of PAPP-A in OHSS risk group of women undergoing IVF stimulation]. Ceska Gynekol. 2009;74(2):80-4.

93. Sahin N, Toylu A, Gulekli B, Dogan E, Kovali M, Atabey N. The levels of hepatocyte growth factor in serum and follicular fluid and the expression of c-Met in granulosa cells in patients with polycystic ovary syndrome. Fertil Steril. 2013;99(1):264-9 e3.

94. Honda T, Fujiwara H, Yoshioka S, Yamada S, Nakayama T, Egawa M, et al. Laminin and fibronectin concentrations of the follicular fluid correlate with granulosa cell luteinization and oocyte quality. Reprod Med Biol. 2004;3(1):43-9.

95. Silvan G, Illera JC, Illera M. Determination of follicular fluid estradiol levels by enzyme-linked immunosorbent assay. Steroids. 1993;58(7):324-9.

96. Tas F, Bilgin E, Tastekin D, Erturk K, Duranyildiz D. Clinical significance of serum laminin levels in patients with lung cancer. Biomed Rep. 2016;4(4):485-8.

97. Arici A, Matalliotakis I, Goumenou A, Koumantakis G, Fragouli Y, Mahutte NG. Increased pregnancyassociated plasma protein-A (PAPP-A) concentrations in peritoneal fluid of women with endometriosis. Am J Reprod Immunol. 2003;49(2):70-4.

98. Zong LL, Li YL, Ha XQ. Determination of HGF concentration in serum and peritoneal fluid in women with endometriosis. Di Yi Jun Yi Da Xue Xue Bao. 2003;23(8):757-60.

99. Khan KN, Masuzaki H, Fujishita A, Kitajima M, Hiraki K, Miura S, et al. Peritoneal fluid and serum levels of hepatocyte growth factor may predict the activity of endometriosis. Acta Obstet Gynecol Scand. 2006;85(4):458-66.

100. Siddiqui RA, Kochhar R, Singh V, Rajwanshi A, Goenka MK, Mehta SK. Evaluation of fibronectin as a marker of malignant ascites. J Gastroenterol Hepatol. 1992;7(2):161-4.

101. Kauma S, Clark MR, White C, Halme J. Production of fibronectin by peritoneal macrophages and concentration of fibronectin in peritoneal fluid from patients with or without endometriosis. Obstet Gynecol. 1988;72(1):13-8.

102. Byers LJ, Osborne JL, Carson LF, Carter JR, Haney AF, Weinberg JB, et al. Increased levels of laminin in ascitic fluid of patients with ovarian cancer. Cancer Lett. 1995;88(1):67-72.

Table 1. Concentration gradients of representative hormones, growth factors, and extracellular matrix proteins in the FF, PF, and serum.

Table Table 1. Concentration gradients of representative hormones, growth factors, and extracellular matrix proteins in t

Figure legends 


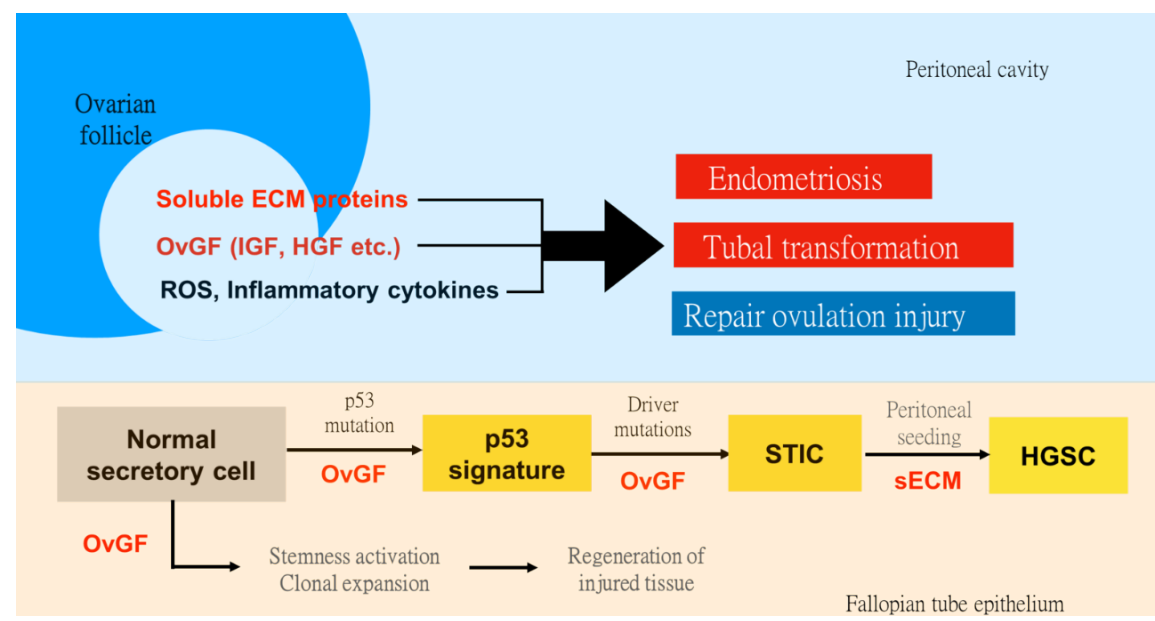

Figure 1. Growth and metastasis-promoting activities of the ovulatory follicular fluid in the peritoneal cavity supportsmalignant transformation, tissue regeneration, and the development of endometriosis. High levels of growth factors in the ovulatory follicular fluid (OvGF), such as the IGFaxis proteins and hepatocyte growth factor (HGF), are responsible for the regeneration of injured tissue caused by ovulation and the stepwise malignant transformation of the fimbrial epithelial cells. The stepwise transformation is initiated with p53 mutation and progression to serous tubal intraepithelial carcinoma (STIC); STIC-driver mutations accumulate upon repeated exposure to mutagenic reactive oxygen species (ROS) and inflammatory cytokines in the follicular fluid (FF). With the help of soluble extracellular proteins in FF, the STIC cells metastasize to the peritoneum lining the ovary, leading to widespread high-grade serous carcinoma. The same mechanism may also promote the survival, attachment, growth, and peritoneal spreading of endometrial cells in endometriosis.

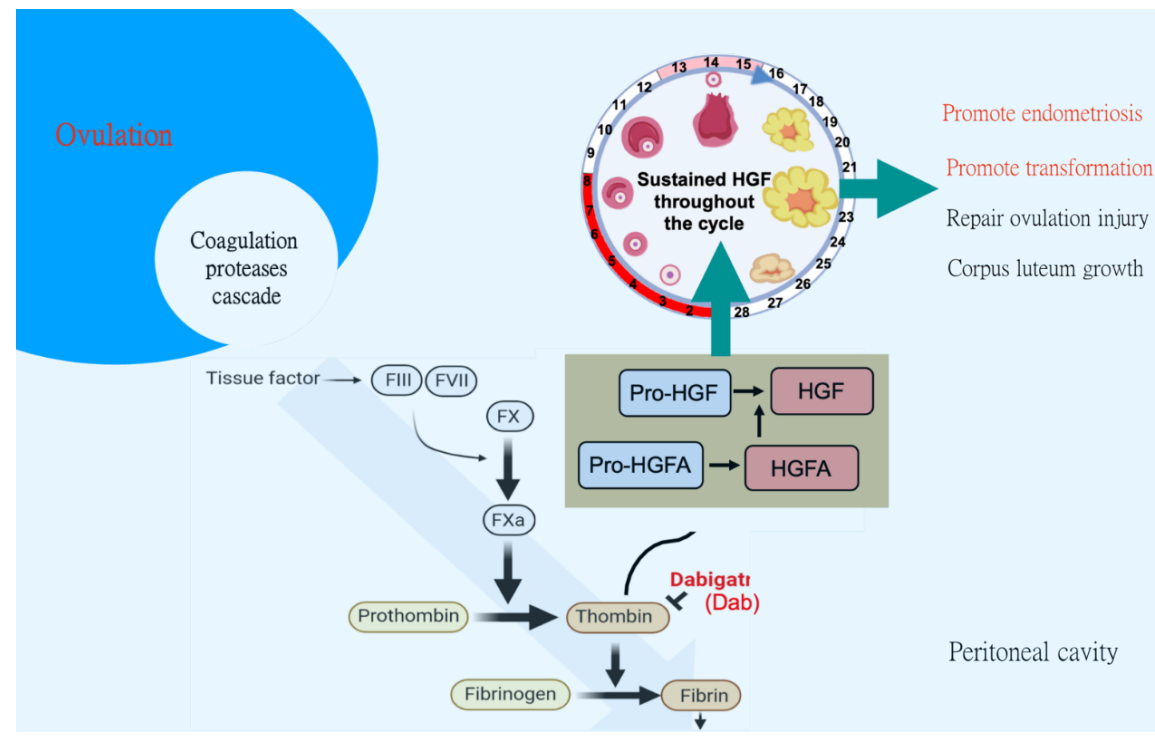

Figure 2. The coagulation cascade/HGF machinery in the follicular fluid exhibits prolonged growth-related activity that may promote the genesis of endometriosis. The ovarian follicular fluid (FF) exhibits prolonged transformation- and regeneration-related activities after mixing with the peritoneal fluid (PF). The coagulation cascade and the hepatocyte growth factor (HGF) are responsible for this 
prolonged activity. High levels of the coagulation cascade proteases, the downstream pro-HGF activator (pro-HGFA), and the HGF in the FF and PF ensure a constant supply of active-HGF, lasting throughout the ovulation cycle. This prolonged HGF activity promotes the proliferative repair of the ovarian surface and fallopian tube fimbrial epithelia as well as the growth of the corpus luteum. The same activity also inadvertently promotes the malignant transformation of the fimbrial epithelium, and the development of endometriosis.
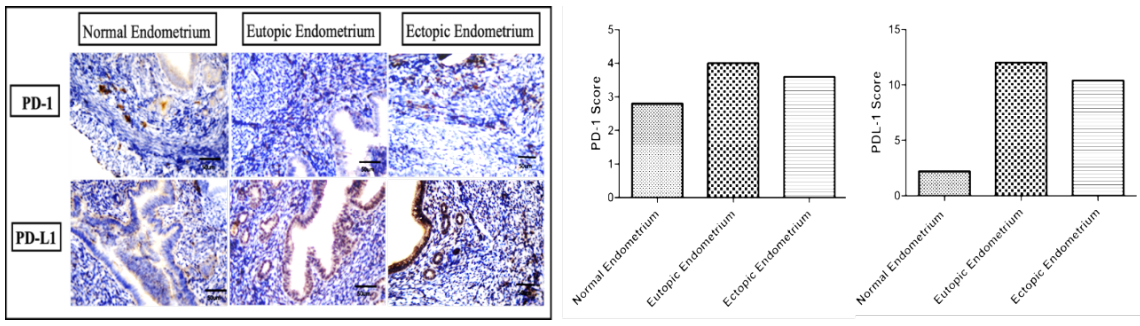

Figure 3. An example of overexpression of PD-L1 in endometriosis. Immunohistochemistry of PD-1 and PD-L1 in the eutopic endometrium and ectopic endometrium (endometriotic lesions) of an endometriosis patient and in the endometrium of a healthy woman is shown. The immunoreactive scoring, in which a product of the IHC staining intensity $(0-3)$ and the percentage of positively stained cells $(0,0 \%$; 1, 1-10\%; 2, 11-50\%; 3, 51-80\%, and 4, [?] 80\%) was calculated, and showed in the right panel.

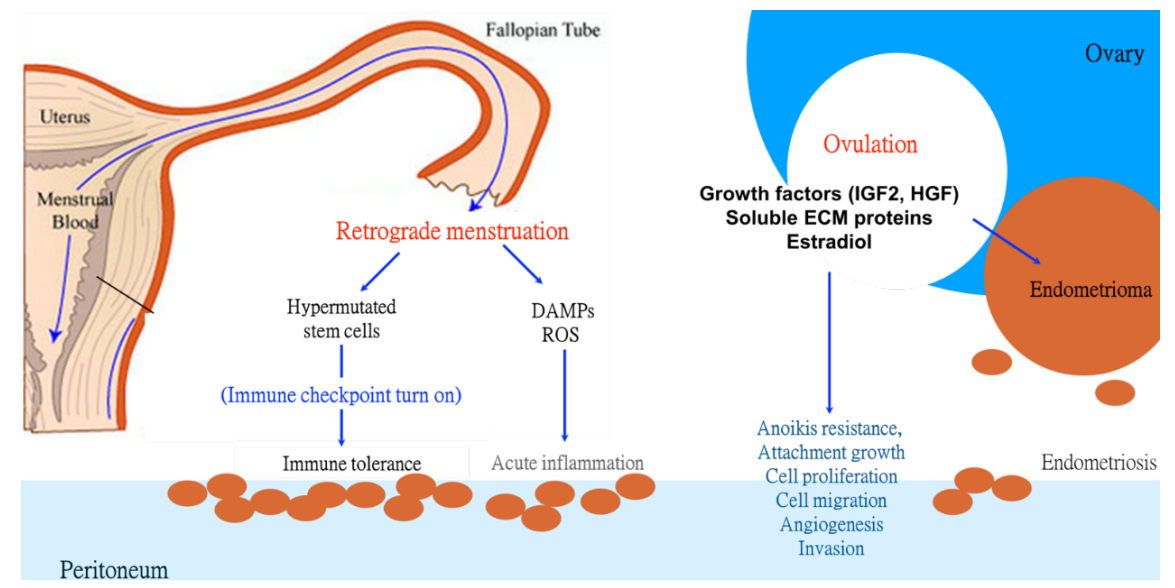

Figure 4. The double engines and single checkpoint theory of the genesis of endometriosis. Retrograde menstruation is the source of stem cells from the broken-down endometrial tissue. It supplies immunogenic and tissue-damaging substances, such as damage-associated molecular patterns (DAMPs) and reactive oxygen species (ROS), to trigger acute inflammation in endometriosis. Ovulation is the other engine that drives the development of endometriosis. Fueled by components that drive malignant transformation, such as growth factors, soluble extracellular matrix (sECM) proteins, and estradiol in the ovulatory follicle, ovulation can induce or augment activities related to malignant transformation, such as anoikis resistance, attachment growth, cell proliferation, migration, and invasion, as well as angiogenesis. Thus, it aids the development of endometriosis. Meanwhile, the endometriotic cells inherit the hypermutated genome from the stem cells present in the eutopic endometrium. By up-regulating the PD-1/PD-L1 immune checkpoint blockade, the progenies acquire immune tolerance for long-term survival and growth at ectopic locations. 